\title{
Inflammation and cardiovascular risk
}

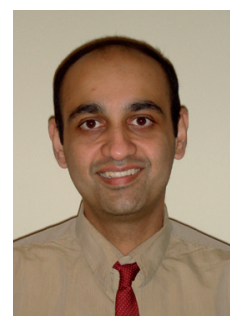

Vijay Nambi speaks to Christine Forder, Commissioning Editor

Vijay Nambi

Section of Atherosclerosis \& Vascular Medicine, Baylor College of Medicine, Center for Cardiovascular Prevention, The Methodist DeBakey Heart \& Vascular Center and Ben Taub General Hospital and Michael E DeBakey Veterans Affairs Hospital, Houston, TX, USA = Tel. : +1 7137985800 = Fax: +1 7137987885 =vnambi@bcm.tmc.edu

Vijay Nambi is an Assistant Professor in the Section of Atherosclerosis and Vascular Medicine, Baylor College of Medicine, Center for Cardiovascular Prevention, the Methodist DeBakey Heart Center, Staff Cardiologist at the Ben Taub General Hospital and Staff at the Michael E DeBakey Veterans Affairs Hospital in Houston, TX, USA. He also serves as the program director of the Atherosclerosis and Vascular Medicine fellowship at the Baylor College of Medicine. His research interests are biomarkers, risk stratification and imaging as related to coronary and peripheral arterial disease.

- How did you become involved in the field of atherosclerosis and vascular medicine?

My fascination with the field began during my residency days when I started to understand how early the process of atherogenesis begins in humans. My fascination only grew realizing how ubiquitous the process was and how there was variable manifestation of the disease with some individuals having focal disease while others having a diffuse disease. I felt that to understand the disease well, training in both vascular medicine and cardiology would be important. I was fortunate enough to be able to train in two great institutions and under a leader in the field, Dr Christie Ballantyne.

Could you briefly explain the role that early identification, of those at risk of atherosclerotic disease, plays in patient management and treatment?

Clearly, therapies we currently have can decrease the risk of the most feared complications of atherosclerosis, namely myocardial infarction, stroke and death. Early identification of at-risk individuals will allow us to institute preventive strategies early on and thereby help prevent these events.

\section{- How big a role does inflammation play in cardiac risk and how can individuals reduce this risk?}

Data now clearly suggest that inflammation plays a major role in the various stages of atherogenesis. Elevated high-sensitivity C-reactive protein (hs-CRP) levels have been associated with coronary heart disease (CHD), cerebrovascular disease and incident $\mathrm{CHD}$ and stroke.
Furthermore, chronic inflammatory conditions, such as rheumatoid arthritis, have now been shown to be associated with an increased risk for CHD. There have been no therapies that had targeted 'inflammation' for CHD risk prevention until recently. The Justification for the Use of Statins in Prevention: an Intervention Trial Evaluating Rosuvastatin (JUPITER) study most recently demonstrated that the treatment of individuals with LDL-cholesterol (LDL-C) levels of less than $130 \mathrm{mg} / \mathrm{dl}$ and hs-CRP of greater than $2 \mathrm{mg} / \mathrm{l}$ with rosuvastatin was associated with a significant decrease in the risk of incident cardiovascular events. Further studies on agents that target inflammation for prevention of cardiovascular disease (CVD) will be required in the future.

6...chronic inflammatory conditions, such as rheumatoid arthritis, have now been shown to be associated with an increased risk for CHD."

\section{- Do you see these new promising} biomarkers becoming part of routine screening in already known risk groups?

Several exciting, novel biomarkers have been identified and will continue to be identified. For any novel biomarker to have clinical utility they must either be of value in risk prediction or as a target of therapy. CRP has been by far the most advanced of the biomarkers. It has demonstrated consistent association with CVD, has been shown to be of value in risk prediction beyond traditional risk-prediction scores and now the JUPITER study has demonstrated that

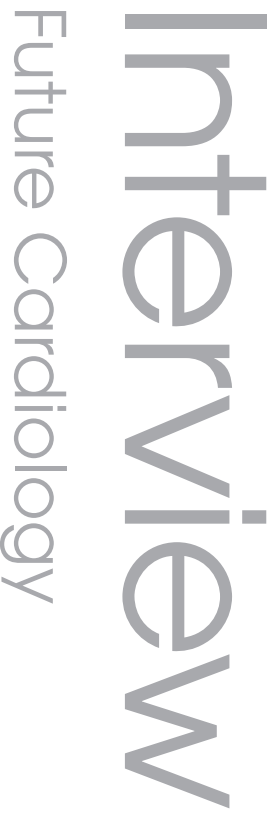

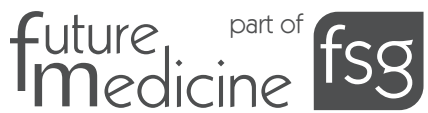


treating those individuals with elevated hs-CRP with statins can lead to a decreased incidence of CVD events. Among the other biomarkers, lipoprotein-associated phospholipase $A_{2}$ $\left(\right.$ LpPLA $\left._{2}\right)$ seems to have a lot of promise and novel agents that target LpPLA 2 are currently under study. Other biomarkers such as lipoprotein (a) and homocysteine have been associated with CHD, but therapy to lower homocysteine with folic acid has not demonstrated any benefits. Therefore, I do see some of the biomarkers being used more in screening, but most likely in selected populations such as those who are intermediate in risk where further risk stratification would help. With advances in technology, if further cost-effective methods are identified, one could foresee a panel of markers being used in risk stratification. One would hope that several of these could become potential targets for therapy.

66

"...combination of imaging, biomarkers and genetics may in concert offer our best option to stratify risk and allow us to personalize care."

- Do new biomarkers have the potential to play as big a role as those already identified and routinely screened for $\mathrm{C}$-reactive protein? Absolutely, although traditional risk scores do a great job in the prediction of CVD, they have limitations. The majority of the events happen in those who are low and intermediate risk as they form the majority of the population. Hence, there is a need to better stratify risk. Similarly, although we have made significant progress in the management and prevention of CVD, coronary artery disease and strokes are still the leading causes of mortality in the western world. Therefore, other new therapeutic options are also required. Biomarkers can play a major role in both risk stratification and as a target of therapy.

\section{- Your recent study suggests the involvement of multiple biomarkers (hs-CRP and LPPLA ${ }_{2}$ ) for risk stratification, how could the use of ultrasound fit in with this to further identify the risk?}

In our study, we used a combination of hs-CRP and LpPLA ${ }_{2}$ to see if it can improve stroke-risk prediction beyond traditional risk factors. We found that the addition of LpPLA 2 and hs-CRP improved the area under the receiver operator characteristic curve and reclassified the risk group of several individuals. However, as was evident from our data, there is still much room to improve risk prediction. Other than biomarkers, imaging and genetic markers can be used to improve risk prediction. Imaging tests, such as ultrasound, directly visualize atherosclerosis and, therefore, may have added value in risk prediction. In fact, the combination of imaging, biomarkers and genetics may in concert offer our best option to stratify risk and allow us to personalize care.

\section{- How does risk stratification related to the management and treatment of patients (i.e., what does low/intermediate/high risk) translate to as a treatment modality?}

Estimated risk has been used to guide intensity of therapy, for example the LDL-C goals. Obviously, individuals who are at higher risk for CHD events will benefit from more aggressive risk modification therapies and will require them to be instituted early on. Data that we presented at the American Heart Association 2008 Scientific Sessions demonstrated that in the Atherosclerosis Risk in Communities (ARIC) study among the low- and intermediate-risk individuals, there is in fact a spectrum of risk and that it is possible to identify these individuals with imaging tests such as carotid intima-media thickness. However, if you look at risk factors, it is the LDL-C levels whose targets change the most based on estimated risk.

\section{- How do you see the use of biomarkers for the identification of novel therapeutic targets advancing in the next 5 years?}

I think more biomarkers and their association with CVD will be described. In this, the '-omic' era with increasing throughput, proteomics, metabolomics and genomics will clearly play a major role in identifying the markers with the most promise. Any new marker in the causal pathway of atherothrombosis would become obvious targets for the development of therapy.

\section{What is needed to further research and improve understanding in the area of arterial disease and atherosclerosis?}

I think all facets of atherogenesis and its manifestations need further research. We have advanced in leaps and bounds over the last few decades in understanding some key steps and processes involved in atherogenesis and in the treatment of acute cardiovascular syndromes. However, while we are able to identify high-risk individuals, we are still unable to predict why and 
when an acute event would occur. Furthermore, we still do not know why some individuals have a multibed atherosclerosis while others have a single vessel, single territory atherosclerosis and, yet, others have no atherosclerosis. There is clearly a lot more work left to be done.

\section{- How do you see cardiac imaging techniques advancing in the next 5 years?}

Technological advances are really pushing imaging techniques forward. Currently, molecular imaging of specific targets of the atherosclerotic plaque is being investigated with a focus on identifying the rupture prone vulnerable plaque. Another important area that has received a lot of attention is in fusion imaging. We recently described a way to register ultrasound and MRI using some novel technology. These kinds of fusion imaging will allow to us to leverage the best that each technology has to offer. Eventually these may help in identification of the vulnerable plaque and, furthermore, may offer novel methods to deliver therapy.

\section{Where do you think your research interests will be focused in the next 5 years?}

My research interests are primarily centered on two areas: cardiovascular risk stratification and peripheral arterial disease (PAD). I have interest in the use of biomarkers and imaging, specifically ultrasound, with respect to cardiovascular risk stratification. Now the other area of my interest is in PAD - the most aggressive and most ignored form of atherosclerosis, as you know. Individuals with PAD have among the highest rates of cardiovascular events and at least two out of three of them have atherosclerosis of multiple arterial beds. We are looking at the use of biomarkers in the prediction of outcomes in individuals with PAD.

\section{Financial \& competing interests disclosure}

Vijay Nambi has received grant funding from the Gillson Longenbaugh Foundation and Gulf Coast Medical Foundation. The author has also received a research grant from the Northwestern Cardiovascular Young Investigators forum as a 2nd place winner in clinical research. The author has no relevant affliations or financial involvement with any organization or entity with a financial interest in or financial conflict with the subject matter or materials discussed in the manuscript. This includes employment, consultancies, honoraria, stock ownership or options, expert testimony, grants or patents received or pending, or royalties.

No writing assistance was utilized in the production of this manuscript.

\section{Affiliation}

- Vijay Nambi

Section of Atherosclerosis \& Vascular Medicine, Baylor College of Medicine, Center for Cardiovascular Prevention, The Methodist DeBakey Heart \& Vasular Center,

Houston, TX, USA

and,

Staff Cardiologist, Ben Taub General Hospital, Houston, TX, USA

and,

Staff, Michael E DeBakey Veterans Affairs

Hospital, Houston, TX, USA

Tel.: +1 7137985800

Fax: +17137987885

vnambi@bcm.tmc.edu 